\title{
Reinforcement of Na-Alginate Based Films with Carrot Juice Processing Wastes
}

Hulya Cakmak ${ }^{1}$ (D) Fatma Unal ${ }^{2}$ (D)

${ }^{1}$ Hitit University, Department of Food Engineering, Corum, Turkey

${ }^{2}$ Hitit University, Department of Metallurgical and Materials Engineering, Corum, Turkey

\section{A B S T R AC T}

Tn this study, Na-alginate based films were produced with the addition of cellulosic fibres from carrot juice processing wastes for employment of agricultural wastes as reinforcing agent in biobased films for food packaging purposes. These films were characterized by water vapour permeability (WVP), colour, XRD, transmittance and the SEM analysis. The WVP of the Na-alginate based films were significantly decreased upon the addition of carrot fibre at each level of incorporation ( $1 \%$ and $5 \%$, w/w on alginate basis). Depending on the colour values, the obtained films were highly transparent, but the yellowness of the $5 \%$ carrot fibre film (CFF) was significantly higher than control ( $0 \%$ CFF) and $1 \%$ CFF samples $(p<0.05)$. The transmittance of control film was higher than the carrot fibre added films, since the lower light impermeability of $5 \%$ CFF was notable in the visual observations and the SEM images. The results revealed that the obtained carrot fibre cellulosic material may be used as reinforcing agent in biobased films for food packaging applications.

Keywords:

Carrot pulp; Alginate; Edible film; Food packaging; Waste.

\author{
Article History: \\ Received: 2020/03/20 \\ Accepted: 2020/06/30 \\ Online: $2020 / 09 / 30$
}

Correspondence to: Hulya Cakmak, Hitit University, Food Engineering, 19030, Corum, TURKEY

E-Mail:hulyacakmak@hitit.edu.tr Phone: +90 3642274333

Fax: +903642274535

\section{INTRODUCTION}

lginate is naturally found in the structu- re of brown microalgae and composed of D-mannuronate and L-guluronate monomers $[1,2,3]$. It is water-soluble and biodegradable polysaccharide that has film-forming ability thus can be utilized in bio-based film formulations for food packing applications $[1,4,5]$. By having a hydrophilic matrix, alginate has a poor resistance to water vapour, therefore either cross linking with the polyvalent ions or reinforcement with cellulosic fibres for improving the water barrier and mechanical properties is recommended in the literature $[1,2,6,7,8]$.

Nanocellulose addition to the Na-alginate based films at 5\% rate improved the WVP and the tensile strength was also increased at the same nanocellulose enrichment level [9]. However, the cellulose fibres and cellulose nanowhiskers that were extracted from mulberry pulp addition to Na-alginate based films had different impact on mechanical and water barrier properties [8]. The cellulose nanowhiskers at each reinforcement level had higher tensile strength and elastic modulus compared to the control film, while the cellulose fibre addition had a negative effects on these mechanical parameters. Both of these cellulosic sources did not impro- ve the water vapour permeability (WVP), however cellulose nanowhiskers reinforced films had significantly lower WVP than the cellulose fibre added films. Similar to this study, Sirviö et al. [10] had studied the effects of nanofibrillated cellulose extracted from birch pulp on the mechanical and water barrier properties of alginate based films. The tensile strength and elastic modulus of the films were improved when compared with the pristine alginate films up to $10 \%$ reinforcement level, but WVP of nanofibrillated cellulose reinforced films were similar with the control film.

Agro-industrial wastes especially from fruit and vegetable processing by-products mainly composed of cellulosic fibres, and recently these wastes are taking part in the biodegradable packaging film formulations as novel and "green" alternatives for mechanical reinforcement and improvement of barrier properties of films [7, $11,12,13]$. For example, seaweed based films that were reinforced with microcrystalline cellulose extracted from bamboo had better mechanical and water barrier properties compared to neat seaweed films [14]. Also, nanofibrillated cellulose extracted from sugar palm wastes were incorporated into the sugar palm starch films, and the tensile strength and modulus values were 
improved with respect to sugar palm fibre addition [13]. In recent studies, carrot pulp was also employed in the biobased pectin or HPMC films in order to improve the mechanical properties of the films as well as functional properties like hydration and antioxidant capacity $[7,12]$.

The aim of this article was to investigate the effect of carrot pulp cellulose addition into the $\mathrm{Na}$-alginate based films on water vapour permeability and characterization of the obtained films was carried out by using spectroscopic (colour), optical (transmittance), morphological (SEM) and crystalline structure (XRD) analysis.

\section{MATERIAL AND METHODS}

\section{Materials}

Na-alginate with 99\% purity (Protanal PH 6160, FMC International, Ireland) was kindly supplied from FMC International. Depending on the specifications given by the supplier, the sample had $12.4 \%$ moisture, $24.7 \%$ d.b. ash, while $99.92 \%$ of alginate powder diameter was smaller than $180 \mu \mathrm{m}$. $\mathrm{HCl}$ (37-38\%, Merck KGaA, Germany), $\mathrm{NaOH}$ (99\%, Tekkim Kimya San. Tic., Bursa), $\mathrm{K}_{2} \mathrm{CO}_{3}$ (99.6\%, JT Baker Chemical Co., Phillipsburg) and glycerin (99.5\%, Smart Kimya Ltd. Sti., Izmir) was supplied from local distributors.

\section{Extraction of Carrot Fibre}

The fresh carrots were washed with tap water and handpeeled. Following the slicing into $3 \mathrm{~cm}$ thickness, the juice and the pulp of carrots were separated by the juice extractor (Arzum Multivit AR 1060, Turkey). The excessive juice remained on the separated pulp was drained with a sieve and it was heat treated at $95^{\circ} \mathrm{C}$ for $5 \mathrm{~min}$ for enzyme inactivation, then air dried at $70^{\circ} \mathrm{C}$ for $8.5 \mathrm{~h}$ in an oven (Memmert, UN 55, Germany). Dried carrot pulp was crushed with a hammer mill (Brabender, SM3, Germany), and the fibres passing through $355 \mu \mathrm{m}$ sieve was used in further extraction step. The cellulosic material extraction from carrot was carried out according to the study of Manzato et al. [15], Sogut and Cakmak [16] with minor modifications.

$8 \mathrm{~g}$ of dried carrot pulp with 1:30 (w/v) of 20\% (w/v) $\mathrm{NaOH}$ was autoclaved at $135^{\circ} \mathrm{C}$ for $30 \mathrm{~min}$ (Alp, CL 40M, Japan). Prior to the centrifugation, the mixture was neutralized using $0.1 \mathrm{M} \mathrm{HCl} .30 \mathrm{ml}$ of this neutralized extract was centrifuged at $20^{\circ} \mathrm{C} 4000 \mathrm{rpm}$ for $2 \mathrm{~min}$ (Sigma 3-30K, Germany). Approximately $20 \mathrm{ml}$ of supernatant was discarded and replaced with tridistilled water, and this step was repeated for 10 times until a clear supernatant was obtained. The insoluble cellulosic precipitate from carrot was dried at $60^{\circ} \mathrm{C}$ for $24 \mathrm{~h}$ in a universal oven (Memmert, UN 55, Germany).

\section{Preparation of Films}

Na-alginate based films were prepared by solvent casting method. $1 \%(\mathrm{w} / \mathrm{w}) \mathrm{Na}$-alginate powder was mixed with distilled water for $3 \mathrm{~h}$ at $65^{\circ} \mathrm{C}$ with a magnetic stirrer (Wisd, MSH20A, Korea). After the mixture was completely homogenized, $25 \%$ (w/w, on alginate basis) glycerol was mixed with the alginate solution for $1 \mathrm{~h}$ at the same conditions. $20 \mathrm{ml}$ of prepared mixture was poured into the PET petri dishes $(\varnothing=90 \mathrm{~mm})$, and then allowed to dry at $40^{\circ} \mathrm{C}$ for $48 \mathrm{~h}$ in an oven (Memmert, UN 55, Germany). The dried films were peeled from the petri dishes and further used in the analysis.

Carrot fibre added films were produced similarly to the control film, $1 \%$ or $5 \%$ (w/w, on alginate basis) carrot fibre was weighed into the beaker that contained distilled water with $\mathrm{Na}$-alginate powder and they were mixed for $3 \mathrm{~h}$ at $65^{\circ} \mathrm{C}$ with a magnetic stirrer (Wisd, MSH20A, Korea). Then the rest of the procedure was repeated.

\section{Water Vapour Permeability (WVP) Measurement}

The WVP of films were measured according to the method of Huang et al. [17] with some modifications. One third of the plastic test cups were filled with distilled water and the specimen was tightly attached to the lid which was open to the testing chamber. The test cups then placed in a desiccator conditioned to $43 \% \mathrm{RH}$ and $25^{\circ} \mathrm{C}$ with saturated $\mathrm{K}_{2} \mathrm{CO}_{3}$ solution. The test cups were weighed with an analytical balance (Precisa Gravimetrics, XB220A, Switzerland) every $1 \mathrm{~h}$ for total of $24 \mathrm{~h}$ in order to determine the mass change depending on the relative humidity difference. The slope of the mass change versus time graph $(\mathrm{g} / \mathrm{h})$ was obtained and WVTR was calculated by dividing the slope to the film area open to the testing environment. WVP (g/kPa.h.m) was calculated according to the following formulae;

$$
W V P=\frac{W V T R x d}{P\left(\mathrm{R}_{c}-\mathrm{R}_{e}\right)}
$$

here $d$ is the thickness of film (m), $P$ is the saturated water vapour pressure $(\mathrm{kPa})$ at $25^{\circ} \mathrm{C}, R_{c}$ is relative humidity inside the test cup and $R_{e}$ is relative humidity inside the conditioned environment of desiccator.

The thickness of the films was measured with a calliper from randomly selected five different points of the films and the averages of them were reported. 
Table 1. Thickness, WVP and colour values of films.

\begin{tabular}{cccccc}
\hline Sample* & $\begin{array}{c}\text { Film thickness } \\
(\mu \mathrm{m})\end{array}$ & $\begin{array}{c}\text { WVP } \\
(\mathrm{g} / \mathrm{kPa} . \mathrm{h} . \mathrm{m})\end{array}$ & $L^{*}$ & $a^{*}$ & $b^{*}$ \\
\hline CF & $44.30 \pm 5.30^{a}$ & $3.777 \times 10^{-6} \pm 8.955 \times 10^{-8 c}$ & $96.82^{b}$ & $-0.24^{b}$ & $2.86^{a}$ \\
$1 \%$ CFF & $46.30 \pm 5.20^{a}$ & $1.961 \times 10^{-6} \pm 9.498 \times 10^{-8 a}$ & $96.68^{b}$ & $-0.28^{a}$ & $2.93^{a}$ \\
$5 \%$ CFF & $53.30 \pm 5.00^{b}$ & $2.235 \times 10^{-6} \pm 1.175 \times 10^{-8 b}$ & $96.00^{a}$ & $-0.22^{b}$ & $3.64^{b}$ \\
\hline
\end{tabular}

${ }^{a-c}$ Different letters in the same column are significantly different $(\mathrm{p}<0.05)$.

${ }^{\star} \mathrm{CF}$ : control film (pure alginate), $1 \%$ CFF: $1 \%$ carrot fibre film, 5\% CFF: $5 \%$ carrot fibre film.

\section{Colour Measurement}

The colour of the films were measured with a spectrophotometer (Konica Minolta, CM3600D, Japan) using CIE $L^{*} a^{*} b^{*}$ colour scale. In this scale, the lightness is represented by L* (0: black, 100: white), while $+\mathrm{a}^{*} /-\mathrm{a}^{*}$ is redness/ greenness, and $+b^{*} /-b^{*}$ is yellowness/blueness. The standard white calibration plate was used as a background for the films [8].

\section{XRD Analysis}

X-ray diffraction (XRD) patterns with monochromatic $\mathrm{Cu} \mathrm{K} \mathrm{K}_{\alpha}$ radiation $(\lambda=0.15406 \mathrm{~nm})$ at $40 \mathrm{kV}$ and $40 \mathrm{~mA}$ were recorded in the $2 \theta$ angle range from $10^{\circ}$ to $90^{\circ}$ with a scan step of $0.05^{\circ}$ using an X-ray diffractometer (Philips, PW3710 XPert Pro, The Netherlands). From using XRD patterns, the average crystallite size calculations were made by using the following Debye-Scherer formula [15];

$$
D=\frac{k \lambda}{\beta_{D} \cos \theta}
$$

where is $k$ is a constant (0.91), $\lambda$ is wavelength of the X-ray source $(0.15406 \mathrm{~nm}), D$ is average crystallite size, $\beta_{D}$ is the peak width at half-maximum intensity and $\theta$ is the peak position.

Crystallinity index (CI) values of the samples were calculated using the following formula after the peak areas were measured from the peak fitting [18];

$$
C I \%=\left(\mathrm{A}_{\text {crystalline }} / \mathrm{A}_{\text {total }}\right) \times 100
$$

where $A_{\text {crystalline }}$ is the area of crystalline diffractogram and $A_{\text {total }}$ is the total area of the original diffractogram.

\section{Transmittance of The Films}

Transmittance spectra of the films between 300-800 nm with $1 \mathrm{~nm}$ step size were recorded by using UV-Vis-NIR spectrophotometer (Shimadzu, UV-3600 with an integrated ISR 3100 sphere attachment, Japan).

\section{Film Morphology}

The surface of the films and the carrot fibre were evaluated with a scanning electron microscope (Quanta 450
FEG, Oregon, USA) under low vacuum conditions at 2 $\mathrm{kV}$ accelerating voltage. The samples were sputter coated with gold for $60 \mathrm{sec}$. at $5 \mathrm{~mA}$ sputter current, and the film sample was mounted on the sample holder with doublesided tape. The images were recorded between the magnification levels of $1500 \times$ to $10000 \times$.

\section{Statistical Analysis}

The statistical differences between the obtained results were compared with SPSS vers. 16.0 (SPSS Inc., USA) with one-way ANOVA Duncan multiple comparison test at $95 \%$ significance level.

\section{RESULTS AND DISCUSSION}

\section{Water Vapour Permeability}

The WVP of control and carrot fibre added films were given in Table 1. The neat alginate film (CF) had the highest WVP, while the incorporation of $1 \%$ and $5 \%$ carrot fibre improved the WVP significantly $(\mathrm{p}<0.05)$. The polysaccharide based films, especially alginate has a hydrophilic nature, and therefore the WVP of the alginate films were not comparable to the petroleum based plastic film counterparts [10]. However, the addition of carrot fibre into $\mathrm{Na}$-alginate matrix enhanced the neat film structure against the water vapour diffusion by creating a barrier to the water molecules that were trapped in the matrix [19]. Similar results were observed in the literature, the micro cellulose or nanocellulose fibre addition to the neat alginate films decreased the WVP by creating a tortuous pathway to the water molecule transport $[8,9,10,14,16]$. Both of the filler contents acted as a barrier against water vapour transport; however the WVP of $1 \%$ CFF were significantly lower compared to the 5\% CFF independent of the average film thickness (Table 1). The reduction of WVP with added fillers is associated with well dispersion of filler inside the matrix, although there is a limit for addition of cellulosic filler [6]. Over this limit, the filler may be agglomerated inside the matrix which leads to the stagnation of WVP reduction or even cause an increase the water vapour transmission $[6,9,16]$. Therefore, higher WVP of $5 \%$ CFF might be associated with the agglomeration of carrot fibre inside the matrix. 


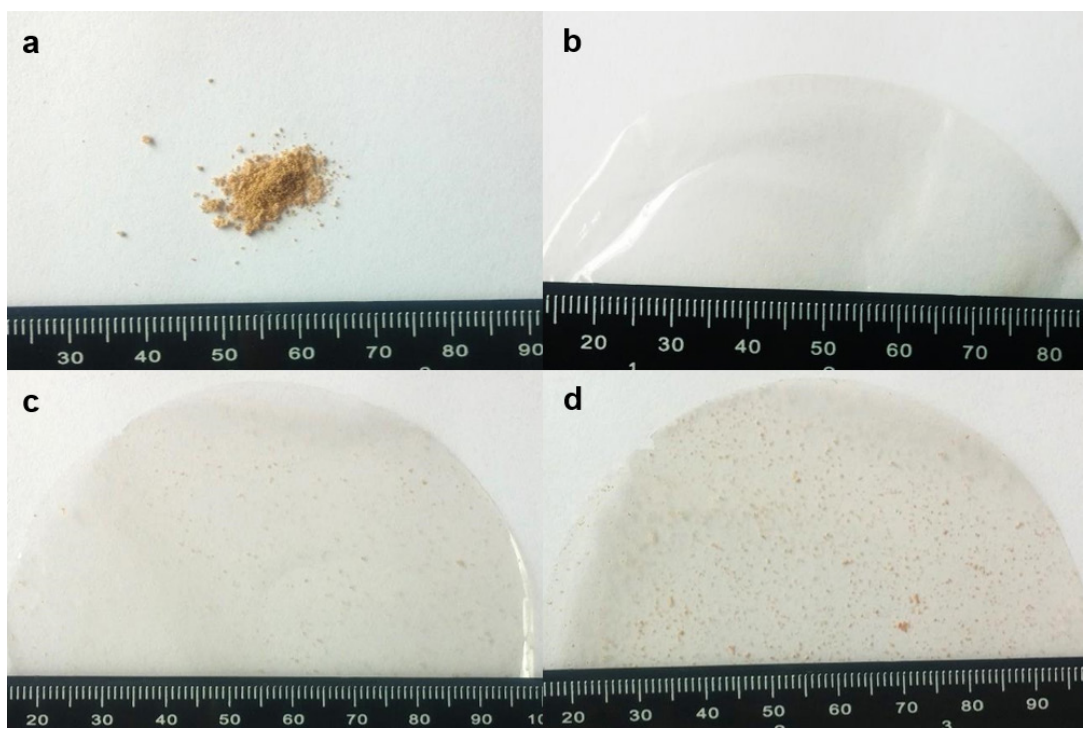

Figure 1. The images of a: carrot fibre, b: CF, c. 1\% CFF, d. $5 \%$ CFF samples.

\section{Colour of The Films}

The surface colour values of the films are given in Table 1 . The $L^{*}$ values that are close to 100 represents the highest transparency, therefore the lightness observation is crucial for film transparency observation. Besides, the incorporation of cellulosic fibre at micro or nano scale may increase the haze and decrease the transparency regardless of source of cellulosic fibre or the type of matrix $[10,19,20]$. L* of the films were quite closer to the 100 , and highly transparent with respect to their visual appearance (Fig. 1), while the 5\% CFF had the lowest lightness compared to neat alginate and $1 \% \mathrm{CF}$ films. This could be due to the light hindrance effect of carrot fibres

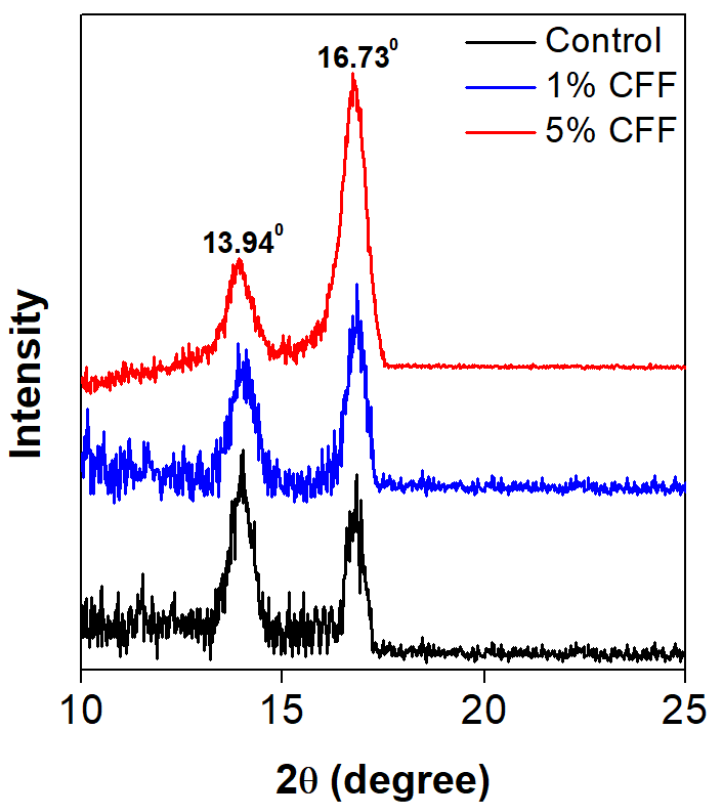

Figure 2. XRD patterns of control film, 1\% CFF and 5\% CFF samples. in the alginate, especially at the $5 \%$ level by possible inhomogeneous distribution of the filler within the matrix [8]. Besides, the highest yellowness was obtained from $5 \%$ CFF $(\mathrm{p}<0.05)$. This observation was also in agreement with the study of Wang et al. [8] upon the mulberry pulp cellulose addition into alginate-based films. However, the yellowness of corn starch-based films with carrot fibre fillers did not increase significantly by increasing the fibre amount from $20 \%$ to $40 \%$ [19]. Therefore, the change in colour values might be related to the colour of the matrix, the source of cellulose, particle diameter and shape of the cellulosic fibre and film production method [7, 8, 19].

\section{XRD and Transmittance of The Films}

The diffraction peaks at $2 \theta \cong 13^{\circ}$ and $16^{\circ}$ were observed in XRD patterns (Fig. 2). The peaks at $13.94^{\circ}$ and $16.73^{\circ}$ were characteristic diffraction peaks of $\mathrm{Na}$-alginate and cellulose, respectively $[9,21,22]$. It was observed that the intensity of the peak around $16^{\circ}$ increased with the increasing the amount of carrot fibre and the peak intensity around $13^{\circ}$ decreased with the increasing amount of carrot fibre. This behaviour indicates that the Na-alginate matrix had a good interaction with the carrot fibre filler.

Crystallinity index values of control, 1\% CFF and 5\% CFF films were $32 \%, 41 \%$ and $45 \%$ respectively. Crystallite size values obtained by Debye-Scherer formula were calculated as 9, 10 and $12 \mathrm{~nm}$ for control film, 1\% CFF and 5\% CFF samples, respectively. The addition of carrot fibre and the increment of the fibre amount in the film resulted in an improvement of crystallinity. Increased crystallinity is important for the packaging films, since it provides more permeability to water vapour and other gases $[10,11]$. It was observed that the water vapour permeability values were in 


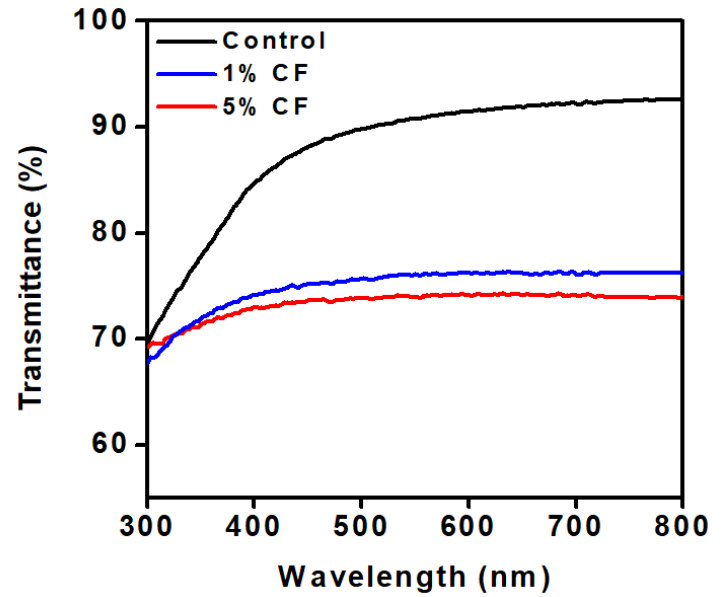

Figure 3. Transmittance curves of control film, 1\% CFF and 5\% CFF samples.

accordance with the crystallinity results. The water vapour permeability values decreased with the increasing crystallinity due to the increment of fibre content in the film.

Transmittance versus wavelength values was given in Fig. 3. The optical transmittance value at $800 \mathrm{~nm}$ of the films decreased from $92.7 \%$ to $74.1 \%$ by increasing the carrot fibre content from $0 \%$ to $5 \%$ wt. The loss in optical transmittance value may be due to the reflection and/or scattering of light at the interface of the fibres. As the amount of fibre in the structure increases, the optical transmittance value decreases as expected; this indicates that the incoming light is reflected and/or scattered more because the interface was increased. Transmittance values were compatible with previous studies, transmittance values of composite films reinforced with the modified ramie fibres and composite films with microcrystalline cellulose particles decreased with increasing fibre/particle content because of the light reflected and/or scattered at the interface $[17,21]$.

\section{Surface Morphology of The Films}

SEM images of carrot fibre and the films were given in Fig. 4. There are no cracks or voids found on the surface of the film samples. In accordance with the visual observations of the films (Fig. 1), the surface of $5 \%$ CFF sample (Fig. 4d) had some irregularities and roughness. The inhomogeneous particle diameter distribution was also present in the images of both the fibre and the fibre added samples. There are also some micron level bubbles visible on the surface of each film; however the surface of control film had more homogeneous structure without any observable porosity. Depending on the addition of carrot fibre especially at the highest level, homogeneous dispersion of fibre might be hindered $[6,9,19]$. The more homogeneous structure of $1 \%$ CFF sample was also confirmed by the results of WVP, that the well dispersed particles inside the alginate matrix had limited the water vapour permeability [19].
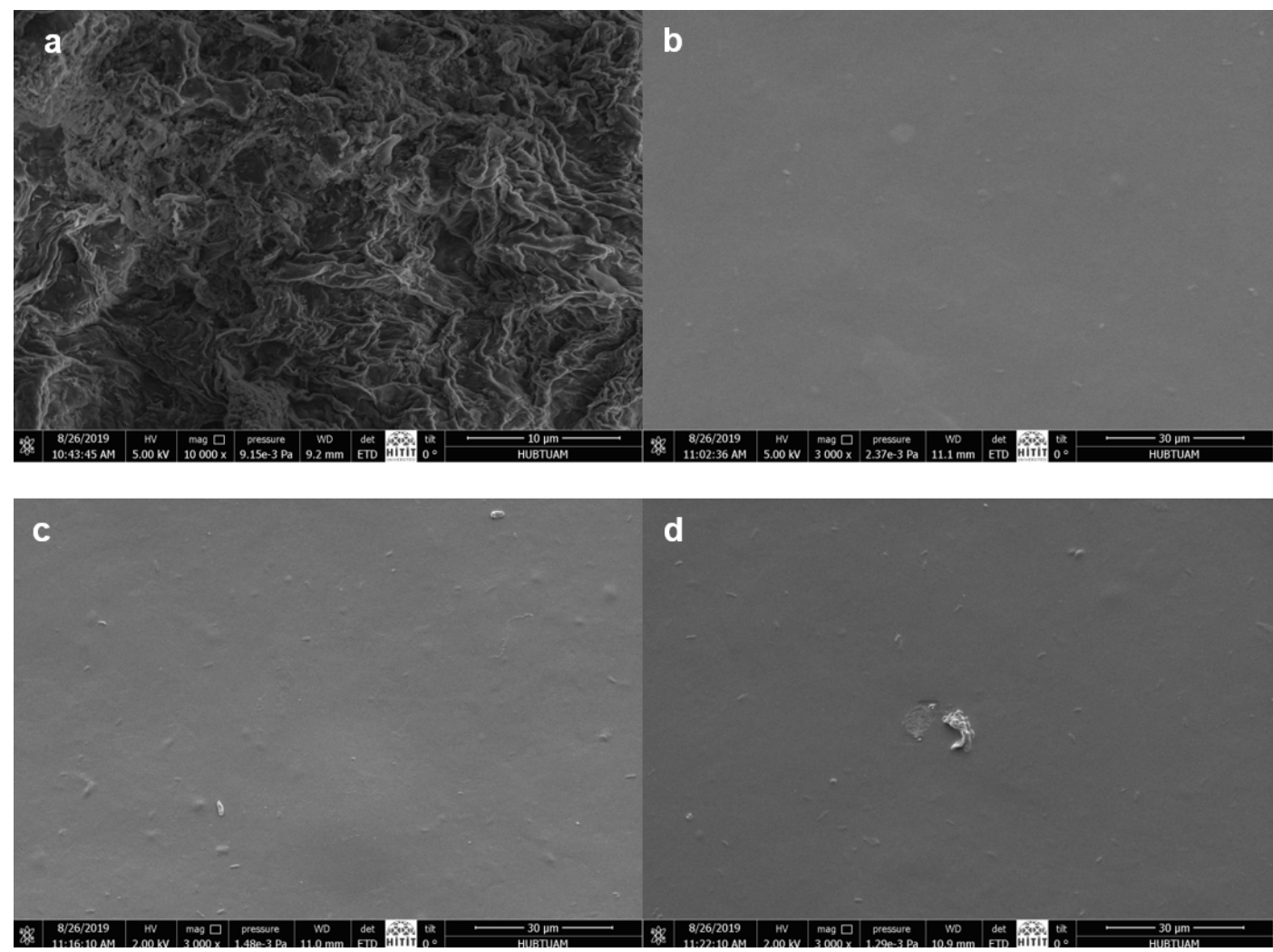

Figure 4. SEM micrographs of (a) carrot fibre at 10000 x scale, (b) CF, (c) $1 \%$ CFF and (d) $5 \%$ CFF at $3000 \times$ scale. 


\section{CONCLUSION}

The current efforts in food packaging application are based on decreasing the environmental burden of plastic films. Completely bio-based films are characterized and further developed as sustainable and environmental friendly alternatives of petroleum based food packaging materials. Therefore, this study is mainly focused on utilizing the carrot juice processing residues as cellulosic filler in the alginate-based films.

The addition of the carrot fibre into the $\mathrm{Na}$-alginate based films improved the film structure against the water vapour permeability; however $5 \%$ (w/w, on alginate basis) CF films had higher WVP compared to $1 \%(\mathrm{w} / \mathrm{w})$ carrot fibre films. The colour and the transmittance values of the 5\% CFF were significantly different from the control and $1 \%$ CFF ( $p<0.05)$. Depending on the SEM images, each film had no cracks or pores on the surface, besides there were some irregularities visible on the $5 \%$ CFF sample depending on the variability of particle diameter distribution of carrot fibre and possible agglomeration behaviour of fibre at $5 \%$ level.

These findings show us that the carrot juice processing wastes as cellulosic fibre resource had a potential to be used in biobased films by enhancing the film properties. However, further studies may focus on mechanical fractionation of the obtained carrot fibres for obtaining much lower particle diameter with homogeneous diameter distribution.

\section{ACKNOWLEDGEMENT}

The authors would like to thank Prof. Dr. Kursat Kazmanli for his help in characterization studies.

\section{References}

1. Maizura M, Fazilah A, Norziah MH, Karim AA. Antibacterial activity and mechanical properties of partially hydrolyzed sago starch-alginate edible film containing lemongrass oil. Journal of Food Science 72(6) (2007) C324-C330.

2. Benavides S, Villalobos-Carvajal R, Reyes JE. Physical, mechanical and antibacterial properties of alginate film: Effect of the crosslinking degree and oregano essential oil concentration. Journal of Food Engineering 110(2) (2012) 232-239.

3. Bierhalz ACK, da Silva MA, Kieckbusch TG. Natamycin release from alginate/pectin films for food packaging applications. Journal of Food Engineering 110(1) (2012) 18-25.

4. Rhim JW. Physical and mechanical properties of water resistant sodium alginate films. LWT-Food Science and Technology 37(3) (2004) 323-330.

5. Tavassoli-Kafrani E, Shekarchizadeh H, Masoudpour-Behabadi M. Development of edible films and coatings from alginates and carrageenans. Carbohydrate Polymers 137 (2016) 360-374.

6. Abdollahi M, Alboofetileh M, Behrooz R, Rezaei M, Miraki R. Reducing water sensitivity of alginate bio-nanocomposite film using cellulose nanoparticles. International Journal of Biological Macromolecules 54 (2013) 166-173.

7. Encalada AMI, Basanta MF, Fissore EN, De’Nobili MD, Rojas AM.
Carrot fiber (CF) composite films for antioxidant preservation: Particle size effect. Carbohydrate Polymers 136 (2016) 1041-1051.

8. Wang LF, Shankar S, Rhim, JW. Properties of alginate-based films reinforced with cellulose fibers and cellulose nanowhiskers isolated from mulberry pulp. Food Hydrocolloids 63 (2017) 201-208.

9. Huq T, Salmieri S, Khan A, Khan RA, Le Tien C, Riedl B, Fraschini C, Bouchard J, Uribe-Calderon J, Kamal MR, Lacroix M. Nanocrystalline cellulose (NCC) reinforced alginate based biodegradable nanocomposite film. Carbohydrate Polymers 90(4) (2012) 1757-1763.

10. Sirviö JA, Kolehmainen A, Liimatainen H, Niinimäki J, Hormi OE. Biocomposite cellulose-alginate films: Promising packaging materials. Food Chemistry 151 (2014) 343-351.

11. Zain NM, Yusop SM, Ahmad I. Preparation and characterization of cellulose and nanocellulose from pomelo (Citrus grandis) albedo. Journal of Nutrition \& Food Sciences 5(1) (2014) 334.

12. Otoni CG, Lodi BD, Lorevice MV, Leitão RC, Ferreira MD, de Moura MR, Mattoso LH. Optimized and scaled-up production of cellulose-reinforced biodegradable composite films made up of carrot processing waste. Industrial Crops and Products 121 (2018) 66-72.

13. Ilyas RA, Sapuan SM, Ibrahim R, Abral H, Ishak MR, Zainudin ES, Atikah MSN, Nurazzi NM, Atiqah A, Ansari MNM, Syafri, E, Asrofi M, Sari HS, Jumaidin R. Effect of sugar palm nanofibrillated cellulose concentrations on morphological, mechanical and physical properties of biodegradable films based on agro-waste sugar palm (Arenga pinnata (Wurmb.) Merr) starch. Journal of Materials Research and Technology 8(5) (2019) 4819-4830.

14. Hasan M, Lai TK, Gopakumar DA, Jawaid M, Owolabi FAT, Mistar EM, Alfatah T, Noriman NZ, Haafiz MKM, Khalil HA. Micro crystalline bamboo cellulose based seaweed biodegradable composite films for sustainable packaging material. Journal of Polymers and the Environment 27(7) (2019) 1602-1612.

15. Manzato L, Rabelo LCA, de Souza SM, da Silva CG, Sanches EA, Rabelo D, Mariuba LAM, Simonsen J. New approach for extraction of cellulose from tucumã's endocarp and its structural characterization. Journal of Molecular Structure 1143 (2017) 229234.

16. Sogut E, Cakmak H. Utilization of carrot (daucus carota 1.) fiber as a filler for chitosan based films. Food Hydrocolloids 106 (2020) 105861 https://doi.org/10.1016/j.foodhyd.2020.105861.

17. Huang $\mathrm{X}$, Xie F, Xiong $\mathrm{X}$. Surface-modified microcrystalline cellulose for reinforcement of chitosan film. Carbohydrate Polymers 201 (2018) 367-373.

18. Park S, Baker JO, Himmel ME, Parilla PA, Johnson DK. Cellulose crystallinity index: measurement techniques and their impact on interpreting cellulase performance. Biotechnology for Biofuels 3(1) (2010) 10.

19. Guimarães IC, dos Reis KC, Menezes EGT, Rodrigues AC, da Silva TF, de Oliveira IRN, Boas EVDBV. Cellulose microfibrillated suspension of carrots obtained by mechanical defibrillation and their application in edible starch films. Industrial Crops and Products 89 (2016) 285-294.

20. Ramesh S, Radhakrishnan P. Cellulose nanoparticles from agroindustrial waste for the development of active packaging. Applied Surface Science 484 (2019) 1274-1281.

21. Yang Q, Lue A, Zhang L. Reinforcement of ramie fibers on regenerated cellulose films. Composites Science and Technology 70(16) (2010) 2319-2324.

22. Alshhab A, Yilmaz E. Sodium alginate/poly (4-vinylpyridine) polyelectrolyte multilayer films: Preparation, characterization and ciprofloxacin $\mathrm{HCl}$ release. International Journal of Biological Macromolecules 147 (2020) 809-820. 